Bundesgesundheitsbl -

Originalien und Ubersichtsarbeiten

V.Thurm · H. Tschäpe

\title{
Gefahrgutrechtliche Voraus- setzungen für den Versand von Diagnostischen Proben, Bakterienkulturen u. a. infektiösen Materialien
}

\section{Zusammenfassung}

Diagnostische Proben (Blut, Organmaterial u.ä.) von Menschen oder Tieren, die Krankheitserreger enthalten, gelten als ansteckungsgefährliche Stoffe und unterliegen beim Transport den gefahrgutrechtlichen Bestimmungen. Das trifft auch auf isolierte Erregerkulturen u.a. infektiöse Materialien zu, die häufig gerade durch medizinische Einrichtungen zu Untersuchungszwecken versandt werden. Der jeweilige Absender, z.B.Arzt oder Laborleiter, trägt dabei eine hohe Verantwortung für die sachgerechte Klassifizierung des Untersuchungsgutes in die zutreffende gefahrgutrechtliche Risikogruppe (in Anlehnung an die Biostoff-V0) und für die dem entsprechende Verpackung und Deklaration der Versandstücke. Der Artikel informiert über die wesentlichen gesetzlichen Bestimmungen und sonstigen Vorschriften, die beim Transport über öffentliche Verkehrswege einschließlich des Postversandes zu berücksichtigen sind, um einen weitestgehenden Infektionsschutz für alle am Transport Beteiligten und die Allgemeinheit zu gewährleisten.

\section{Schlüsselwörter}

Probenversand · Gefahrgutrecht · Infektiöse Materialien · Diagnostische Proben
D. as seit dem 1. Januar 2001 gültige Infektionsschutzgesetz unterstreicht das Anliegen der Bundesrepublik Deutschland, im Interesse des Gemeinwohls und der Gesundheit der Bevölkerung verstärkt gegen Infektionskrankheiten vorzugehen. Zur gezielten Behandlung einzelner Patienten, aber auch zur frühzeitigen Erkennung und Ausbreitungsverhütung von Infektionskrankheiten ist die rasche Untersuchung sowohl Diagnostischer Proben von Erkrankten als auch von isolierten Erregerkulturen für spezielle Aussagen unumgänglich. Ein wichtiges Glied in der Kette zwischen Probeentnahme und Untersuchung der Probe ist ihr Transport. Je unkomplizierter für den Absender, je kostengünstiger für den Kostenträger und je rascher er möglich ist, um so mehr und um so effektiver kann zum Wohle von Patienten und zum Schutz der Allgemeinheit davon Gebrauch gemacht werden.

\section{„Alle Beteiligten müssen dafür sorgen, dass weder aus dem \\ Transport, noch aus der Entnahme und Untersuchung von Proben und Kulturen Infektionsgefahren für Beschäftigte oder die Allgemeinheit erwachsen."}

Auf der anderen Seite ist von allen Beteiligten dafür Sorge zu tragen, dass weder aus dem Transport, noch aus der Entnahme und Untersuchung von Pro- ben und Kulturen Infektionsgefahren für die damit Beschäftigten oder gar die Allgemeinheit erwachsen.

Zahlreiche Anfragen an das Robert Koch-Institut (RKI) gerade aus dem Bereich der medizinischen Einrichtungen in der letzten Zeit zeigen, dass z. T. genereller Informationsbedarf über die beim Transport infektiöser Materialien einzuhaltenden gefahrgutrechtlichen Bestimmungen besteht. Auch führten Unklarheiten in der Interpretation dieser Bestimmungen zu Unsicherheiten, die die praktische Umsetzung behindern oder durch unsachgemäße Verpackung gar zusätzliche Infektionsrisiken schaffen. Beides hat das RKI veranlasst, in Abstimmung mit dem Bundesministerium für Verkehr, Bau- und Wohnungswesen (BMVBW) und - soweit die Postbeförderung solcher Materialien betroffen ist - mit der Deutschen Post AG diesem Informationsdefizit mit dem nachfolgenden Übersichtsartikel zu begegnen.

\section{Grundlegende \\ Rechtsvorschriften}

Zur Gefahrenabwendung beim Transport gefährlicher Güter gibt es in Deutschland und international ein Werk umfangreicher gefahrgutrechtlicher Bestimmungen. Für alle Verkehrsträger weltweit gelten als grundlegendes Regelwerk die, ,Re-

Dr. habil. VolkerThurm

Robert Koch-Institut, Bereich Wernigerode,

Burgstraße 37,38855 Wernigerode 
Bundesgesundheitsbl-

Gesundheitsforsch - Gesundheitsschutz

2001 · 44:823- $828 \odot$ Springer-Verlag 2001

\section{V.Thurm · H. Tschäpe}

Requirements for the dispatch of diagnostic specimens, bacterial cultures and other infectious materials under legislation on dangerous goods

\begin{abstract}
Pathogen-containing diagnostic specimens (blood, organ material, etc.) from humans or animals are considered as infectious substances and, during transport, fall under the provisions of legislation on dangerous goods. This also applies to isolated pathogen cultures and other infectious materials which, frequently, are dispatched by medical institutions for examination purposes. The sender, e.g. physician or head of laboratory, is responsible for the correct classification of the test material into the corresponding risk groups as fixed in the regulations on dangerous goods (based on the regulations of biological substances) and for corresponding packaging and labelling of these items. The article provides information on the essential legal regulations and other provisions which have to be taken into account during transport using public transport routes including dispatch by mail in order to guarantee the best possible protection against infection for all persons involved and the general public.
\end{abstract}

\section{Keywords}

Dispatch of samples · Legislation on dangerous goods ' Infectious materials . Diagnostic specimens

\section{Originalien und Übersichtsarbeiten}

commendations on the Transport of Dangerous Goods - Model Regulations“ der Vereinten Nationen [1]. Sie beinhalten u.a. eine Einteilung der verschiedenen gefährlichen Güter (Gase, explosive, entflammbare, radioaktive, toxische Substanzen u.v.m.) in Gefahrgutklassen. Infektiöse Substanzen gehören der UNKlasse 6.2 an. Für die häufigste Form, den Gefahrguttransport über öffentliche Straßen, ist als grundlegendes Regelwerk das Europäische Übereinkommen vom 30. September 1957 über die internationale Beförderung gefährlicher Güter auf der Straße (ADR) einschließlich Anlagen A und B zu berücksichtigen [2], dessen neu strukturierte Fassung am 1. Juli 2001 völkerrechtlich in Kraft tritt. Die nationale Umsetzung erfolgt bislang durch die Gefahrgutverordnung Straße (GGVS [3]), deren novellierte Form als Gefahrgutverordnung Straße und Eisenbahn (GGVSE) gleichfalls am 1. Juli 2001 in Kraft treten soll. Daneben gibt es verkehrsträgerspezifische internationale Regelwerke einschließlich nationaler Umsetzungen durch Verordnungen, Richtlinien und Bestimmungen für den Eisenbahnverkehr (z. B. RID [4]), den Binnenschiffsverkehr (z. B. ADNR [5]), den Seeschiffsverkehr (z. B. IMDG-Code [6]) und den Luftverkehr (ICAO-TI [7] und IATA-DGR [8]) sowie verkehrsträgerübergreifende Vorschriften wie die Gefahrgutbeauftragtenverordnung (GbV [9]).

Zum Schutz vor Infektionen beim Umgang mit ansteckungsgefährlichen Stoffen existiert für den ,Schutz der Arbeitnehmer gegen Gefährdung durch biologische Arbeitsstoffe bei der Arbeit" eine erst kürzlich (27. Juni 2000) vom Europäischen Parlament novellierte Richtlinie 90/679/EWG [10] des Rates. Sie ist mit der, „Verordnung zur Umsetzung von EG-Richtlinien über den Schutz der Beschäftigten gegen Gefährdung durch biologische Arbeitsstoffe bei der Arbeit" (Biostoffverordnung vom 27. Januar 1999 [11]) in nationales Recht überführt worden. Durch die darin enthaltene konkrete Einstufung einzelner biologischer Arbeitsstoffe in Risikogruppen ergibt sich eine Beziehung zum Gefahrgutrecht der Klasse 6.2. Das für den Gefahrguttransport zuständige Bundesministerium für Verkehr, Bau- und Wohnungswesen prüft gegenwärtig, ob diese inhaltliche Beziehung zwischen beiden gesetzlichen Regelwerken auch rechtsverbindlich hergestellt werden kann.

\section{Begriffsdefinitionen}

\section{Ansteckungsgefährliche Stoffe}

Ansteckungsgefährliche (infektiöse) Stoffe der UN-Gefahrklasse 6.2 sind per definitionem solche, von denen bekannt oder (mit einer gewissen Wahrscheinlichkeit) anzunehmen ist, dass sie:

D Krankheitserreger (z. B. Mikroorganismen) enthalten, die bei Menschen oder Tieren infektiöse Krankheiten verursachen (Gefährdungspotential) und

- die auch in der Lage sind, an der Stelle ihres Freiwerdens Krankheiten auf zufällig anwesende Personen oder Tiere zu übertragen (Übertragungsgefahr).

Während nach den Model Regulations der UN und dem ADR Krankheitserreger bisher ausschließlich als Mikroorganismen unterschiedlicher Klassen (Bakterien, Viren, Pilze usw.) definiert sind, müssen nach jüngerem wissenschaftlichen Erkenntnisstand auch andere infektiöse Agenzien hier zugeordnet werden, z. B. Prionen als Erreger der transmissiblen spongiformen Enzephalopathien (TSE).

\section{„Ist eine ansteckungsgefähr- liche Krankheit eines Patienten bekannt oder besteht dies- bezüglich ein begründeter Verdacht, sind beim Versand von Proben die Gefahrgut- vorschriften zu beachten."}

Gibt es dagegen z. B. für die betreffenden Mikroorganismen keine Hinweise auf eine Pathogenität, oder ist beim ungewollten Freisetzen (z. B. durch Transportschäden) keine Infektionsgefahr im o.g. Sinn vorhanden, unterliegen die betreffenden Stoffe bzw. Proben nicht dem Gefahrgutrecht. Das kann z. B. auf Proben zutreffen, die lediglich zur Analyse bestimmter klinisch-chemischer Werte wie Blutfette, Zucker, Cholesterin entnommen und versandt werden. Ist dem verantwortlichen Arzt jedoch eine ansteckungsgefährliche Krankheit eines Patienten bekannt oder hat er zu deren Vorhandensein einen begründeten Verdacht (z. B. aus der Krankengeschichte des Patienten oder aus festgestellten Symptomen), unterliegt die 
Probe den Gefahrgutvorschriften der Klasse 6.2. Beispiele für ansteckungsgefährliche Stoffe im Bereich medizinischer Einrichtungen sind neben Diagnostischen Proben von Patienten mit ansteckenden Krankheiten auch daraus isolierte Erregerkulturen oder zu anderen Zwecken (Referenzmaterial, Forschung) versandte Mikroorganismen sowie sog. biologische Produkte (z. B. bestimmte Diagnostika). Ferner zählen dazu die bei der Probenahme sowie ambulanten und stationären Versorgung von Patienten mit bestimmten ansteckenden Krankheiten anfallenden kontaminierten Geräte (Spritzen u.a.) und sonstigen Abfälle (Tupfer, Exkrete etc.). Für die Handhabung dieser infektiösen Krankenhausabfälle der sog. LAGA-Gruppe C gibt es gesonderte Regelungen [12].

\section{Diagnostische Proben}

Diagnostische Proben sind nach den Bestimmungen des Gefahrgutrechts (ADR, Anlage A, Randnummer 2650) insbesondere Blut, Gewebeproben, Ausscheidungsstoffe (Stuhl, Urin) oder andere Materialien von Menschen oder Tieren, die zu Untersuchungs- oder Forschungszwecken entnommen und befördert werden. Es muss eindeutig klargestellt werden, dass daraus isolierte Erregerkulturen, auch wenn sie z.B. zum Zwecke weiterführender spezialisierter Diagnostik versandt werden, keine Diagnostischen Proben im o.g. Sinne sind. Das trifft auch für zu Forschungszwecken, z. B. als Referenzmaterial, versandte Mikroorganismenkulturen zu, die gesondert nach dem Gefahrgutrecht zu klassifizieren sind.

\section{Einteilung in Risikogruppen}

Ansteckungsgefährliche Stoffe der Klasse 6.2 ADR werden aufgrund ihres Gefährdungsgrades in solche mit hohem Gefährdungspotential (Risikogruppe 3 und 4) und sonstige (weniger) ansteckungsgefährliche Stoffe (Risikogruppe 1 und 2) eingeteilt. Grundlage der Zuordnung sind in Randnummer 2650 ADR (ab 1. Juli 2001 Unterabschnitt 2.2.62.1.1) verbal formulierte Kriterien, zurückgehend auf Festlegungen der WHO in ihrem Laboratory Biosafety Manual [13]. Sie berücksichtigen die $\mathrm{Pa}$ thogenität der Erreger, ihre Infektiosität (Art und Weise und relative Leichtigkeit der Übertragung), Gefahren für einzelne
Individuen (Mensch, Tier) und solche für die Allgemeinheit sowie die Verfügbarkeit von Präventivmaßnahmen und Behandlungsmöglichkeiten.

Risikogruppe 1 umfasst Mikroorganismen, bei denen es unwahrscheinlich ist, dass sie Krankheiten bei Menschen oder Tieren hervorrufen. Sie stellen daher keine oder nur eine sehr geringe individuelle Gefahr und keine oder nur eine sehr geringe Gefahr für die Allgemeinheit dar. Mikroorganismen und diese enthaltende Stoffe unterliegen nicht den Anforderungen des Gefahrgutrechts. Beispiel: Bacillus subtilis, Staphylococcus epidermidis.

Risikogruppe 2 umfasst weniger gefährliche Krankheitserreger mit mäßiger individueller Gefahr und mit geringer Gefahr für die Allgemeinheit. Beispiel: Staphylococcus aureus, Yersinia enterocolitica.

Risikogruppe 3 umfasst gefährliche Krankheitserreger mit hoher individueller Gefahr, aber geringerer Gefahr für die Allgemeinheit aufgrund begrenzter Übertragungsgefahr und vorhandener Möglichkeiten der Vorbeugung und Behandlung. Beispiel: Bacillus anthracis, Yersinia pestis, HIV.

Risikogruppe 4 umfasst die gefährlichsten Krankheitserreger der Klasse 6.2 mit hoher individueller und hoher Gefahr für die Allgemeinheit. Beispiel: Ebola-Virus, Lassa-Virus.

\section{DieZuordnung eines an- steckungsgefährlichen Stoffes zur betreffenden Risikogruppe entscheidet über die anzuwen- denden Vorschriften und Anforderungen bei Verpackung und Transport.}

Hilfreich für den Gefahrgutabsender, der für die Zuordnung des Materials verantwortlich ist, ist zur Orientierung ein Blick in Anhang III der oben zitierten Richtlinie 90/679/EWG (in Form der dem jeweiligen Wissensstand angepassten aktualisierten Fassung). Der Anhang III enthält eine Auflistung der wesentlichsten humanpathogenen Mikroorganismen u.a. Erreger mit entsprechender Zuordnung zu den einzelnen Risikogruppen, erstellt allerdings unter Arbeitsschutzaspekten.

\section{Verpackung und Transport}

Die Zuordnung eines ansteckungsgefährlichen Stoffes zur betreffenden Risikogruppe entscheidet über die anzuwendenden Vorschriften und Anforderungen bei Verpackung und Transport. Verpackungen für Stoffe der Risikogruppe 2 müssen z. B. den Vorschriften des Anhangs A. 5 der Anlage A des ADR (ab 1. Juli 2001 Kapitel 6.1) entsprechen. Für die Risikogruppen 3 und 4 gelten Sondervorschriften für die Verpackung, die bauartgeprüft und besonders gekennzeichnet sein muss (UN-Gefahrgutverpackung für Klasse 6.2, s. Abb.1). Diese Verpackungen sind bei entsprechenden Handelsfirmen und ggf. über Gefahrguttransportunternehmen erhältlich. Jeder Beförderung von Gefahrgütern über öffentliche Verkehrswege ist bis auf bestimmte Ausnahmen ein Beförderungspapier mitzugeben. Außerdem sind besondere Anforderungen an die Beförderungsmittel und die Fahrzeugführer sowie weitere Vorschriften der Anlage B des ADR zu berücksichtigen ( $a b$ 1. Juli 2001 insbesondere Kapitel 5.4 und Teile 8 und 9).Zugelassene Gefahrguttransportfirmen sind darauf ausgerichtet.

Eine Ausnahme betrifft sog. Kleinmengen. Entsprechend Ausnahme Nr. 55 der Gefahrgutausnahmeverordnung (GGAV [14]) in Verbindung mit Randnummer 10 o11 ADR darf bis zum 31. Dezember 2001 für die Stoffe der Beförderungskategorie $2(<300 \mathrm{~kg}$ der weniger ansteckungsgefährlichen Stoffe der Risikogruppe 2) auf das Beförderungspapier und die Anwendung bestimmter Vorschriften der Anlage B ADR verzichtet werden. Eine weitere Ausnahme von den Bestimmungen des ADR stellt die Beförderung Diagnostischer Proben nach der multilateralen Vereinbarung zum ADR Nummer M96 dar.

\section{Multilaterale Vereinbarung M96}

Deutschland hat am 19. Juni 2000 eine im Vorgriff auf internationale Entscheidungen mit dem Vereinigten Königreich (Großbritannien) und der Tschechischen Republik getroffene multilaterale Vereinbarung M96 über die Beförderung von Diagnostischen Proben [15] gezeichnet. Sie beinhaltet in Abweichung von den Gefahrgutvorschriften der Klasse 6.2 folgende Erleichterungen für den Transport von Diagnostischen Proben, von denen 


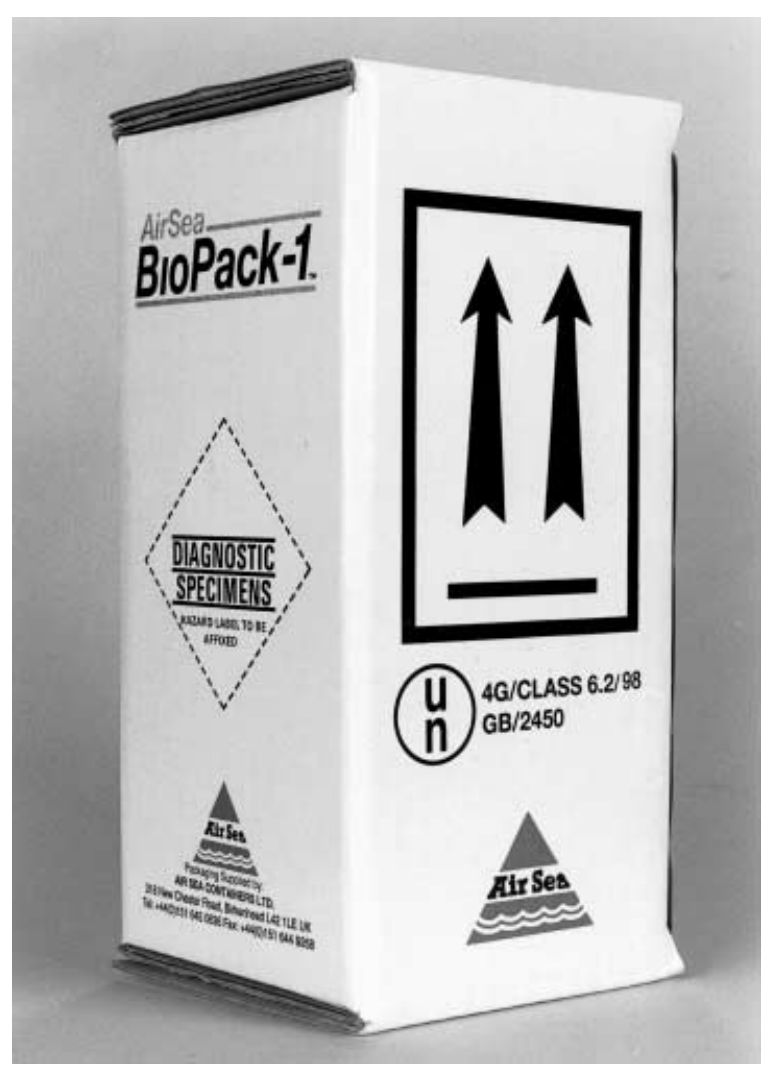

\section{Postversand von infektiösem medizinischen Untersuchungsgut}

Ein großer Teil der Diagnostischen Proben, Bakterienkulturen u.a. infektiösen Stoffe wird durch krankenhaus- oder laboreigene, aber auch durch gewerbliche Kurierdienste über öffentliche Straßen transportiert. Daneben kommt dem Postversand im Inland nach wie vor eine erhebliche Bedeutung zu. Das betrifft insbesondere die Beförderung zu weiter entfernten spezialisierten Untersuchungseinrichtungen, Referenzlaboratorien u.ä.

Die Deutsche Post AG unterliegt ebenso wie Kurier- und Expressdienste oder Spediteure - in Deutschland den Gefahrgutvorschriften für die einzelnen Verkehrsträger, für die Straße damit der GGVS (ab 1. Juli 2001 GGVSE) in Verbindung mit den Anlagen A und B des ADR. Sie kann allerdings - wie andere Dienste auch - Annahmebedingungen vertraglich festlegen und damit den Kreis der zur Beförderung angenommenen Güter einschränken. Davon hat die Deutsche Post AG in ihren „Allgemeinen Geschäftsbedingungen der Deutschen Post AG für den Briefdienst Inland vom 1.10.1999, Regelungen für die Postbeförderung von gefährlichen Stoffen “ [16] sowie in den „Regelungen für die Postbeförderung von medizinischem und biologischem Untersuchungsgut im Inland" (Stand Februar 1999 [17]) Gebrauch gemacht und Einschränkungen festgelegt. Danach können im Briefdienst Diagnostische Proben und Biologische Produkte, die keine ansteckungsgefährlichen Stoffe der Klasse 6.2 enthalten, in Versandverpackungen verschickt werden, die der Europäischen Norm EN 829 [18] entsprechen. Beispiel dafür wären Blutproben für klinisch-chemische Untersuchungen von Patienten ohne ansteckende Krankheiten. Um eine Beschädigung zu vermeiden, sollte die Verpackung jedoch kistenförmig sein.

\section{„Für infektiöses medizinisches oder biologisches Untersu- chungsgut sind bauartgeprüfte Verpackungen vorgeschrieben."}

Für infektiöses medizinisches oder biologisches Untersuchungsgut der Risikogruppe 2, aber auch der RG 1, sind dagegen bauartgeprüfte Verpackungen vorgeschrieben, die nach Randnummer 
Übersicht 1: Verpackungsvorschriften für Diagnostische Proben nach der M96

\section{Grundsatzforderung}

Diagnostische Proben müssen in Verpackungen „Von guter Qualität" verpackt werden, die geeignet sind, allen normalerweise bei der Beförderung auftretenden Belastungen standzuhalten und einen Austritt von Probematerial wirksam zu verhindern.

Ein Versandstück besteht aus drei Verpackungsteilen (s. Abb. 2):

- Die Innenverpackung (Probengefäß)

- Sie enthält das Probematerial und muss flüssigkeitsdicht verschlossen sein. Meist handelt es sich dabei um das bei der Entnahme verwendete praxisübliche Probengefäß (Stuhlröhrchen, Monovette 0. ä.). Es darf nicht mehr als $100 \mathrm{ml}$ enthalten.

- Die wasserdichte zweite Verpackung (Schutzgefäß)

- Das Innengefäß ist in eine wasserdichte 2. Verpackung (handelsüblich: Kunststoffgefäß mit Schraubdeckel) einzusetzen. Bei flüssigen Proben ist zwischen beiden Gefäßen saugfähiges Material in ausreichender Menge anzuordnen. Es können dabei mehrere Innengefäße in die 2.Verpackung eingegeben werden, wenn jedes einzeln mit saugfähigem Material umwickelt ist.

- Die Außenverpackung (Versandhülle)

- Die zwei Verpackungen müssen, durch geeignete Polstermittel (Zellstoff, Polsterfolie) gesichert, nochmals in Außenverpackungen aus stabilem Papier, Pappe, Kunststoff oder Metall verpackt werden. Diese dürfen nicht mehr als insgesamt $500 \mathrm{ml}$ Probenmaterial enthalten.

Kennzeichnung

Jedes Versandstück ist auf der Außenverpackung klar und deutlich mit der Aufschrift „DIAGNOSTISCHE PROBEN“ kenntlich zu machen.

3512 ADR mit dem Symbol UN und einer Codierung gekennzeichnet sind (s. Abb. 1). Sie sind im Fachhandel erhältlich und zusätzlich mit dem Vermerk „Vorsicht infektiös!“ und „Medizinisches Untersuchungsgut“ zu kennzeichnen. Näheres ist den „Regelungen für die Postbeförderung von medizinischem und biologischem Untersuchungsgut Tipps für Einlieferer" der Deutschen Post AG zu entnehmen [17].

In Abstimmung mit der Zentrale der Deutschen Post AG muss darauf hingewiesen werden, dass seit dem 1.10.1999 ansteckungsgefährliche Stoffe der Risikogruppe 3 nicht mehr mit der Deutschen Post AG befördert werden können. Illustriert am konkreten Beispiel bedeutet dies, dass z. B. Sputum-, Stuhloder Blutproben mit begründetem Verdacht auf Tuberkulose, Typhus, Hepatitis oder HIV bzw. Erregerkulturen der Risikogruppe 3 (z. B. Mycobacterium tuberculosis) nicht mit der Deutschen Post AG befördert werden dürfen, sondern dass dafür spezielle für den Gefahrguttransport zugelassene Kurierdienste in Anspruch zu nehmen sind.

Dagegen sind neben den Diagnostischen Proben auch die Mikroorganismenkulturen selbst als Untersuchungsgut für den Versand mit der Deutschen Post AG zugelassen, jedoch auch nur bis zur Risikogruppe 2. Während bei versandten Kulturen die Risikogruppe bekannt ist, ergeben sich bei Diagnostischen Proben häufig Unsicherheiten. So ist in der Praxis durch den für die Risikoeinstufung verantwortlichen Absender, z.B. den die Probe entnehmenden Arzt, und insbesondere bei Erstuntersuchungen oder unklarer Diagnose oft nicht genau vorhersehbar, ob Erreger der Risikogruppe 2 oder 3 in der betreffenden Probe möglicherweise enthalten sind. Dies zieht ggf. eine völlig andere Behandlung, gefahrgutrechtliche Stellung, einen anderen Transportträger und zusätzliche Unsicherheiten und Erschwernisse für die betreffende medizinische Einrichtung nach sich, die notwendige Untersuchungen behindern oder zumindest verzögern können. Das Robert Koch-Institut hat daher angeregt, seitens der Deutschen Post AG zu prüfen, ob bzw. unter welchen Voraussetzungen die erleichternden Bedingungen der M96 (Diagnostische Proben der Risikogruppen 2 und 3) für den Transport als Briefsendung im Inland übernommen werden können.

\section{Gefahrgutbeauftragte und sonstige verantwortliche Personen}

Die Gefahrgutbeauftragtenverordnung (GbV) vom 12. Dezember 1989, die gegenwärtig überarbeitet wird, verpflichtet grundsätzlich Unternehmer und Inhaber von Betrieben, die an der Beförderung gefährlicher Güter beteiligt sind, einen Gefahrgutbeauftragten zu bestellen. Das trifft auch auf Arztpraxen, Krankenhäuser, Laboratorien und sonstige medizinische Einrichtungen zu. Gefahrgutbeauftragter kann eine interne oder externe Person (ggf. der Unternehmer/Inhaber selbst) sein, die jeweils im Besitz eines gültigen Schulungsnachweises der zuständigen Industrie- und Handelskammer ist, im Unternehmen in diese Funktion schriftlich bestellt wurde und die Aufgaben nach der GbV wahrnimmt.

Befreit von der Verpflichtung zur Bestellung eines Gefahrgutbeauftragten nach $\S 1 \mathrm{~b}$ GbV sind - ggf. unter Bedingungen - freigestellte Beförderungen. Dazu zählen auch Beförderungen von Diagnostischen Proben der Risikogruppen 2 und 3 nach der multilateralen Vereinbarung M96 oder der Risikogruppe 2 nach Randnummer 2656 und von Erregerkulturen u.a. ansteckungsgefährlichen Stoffen der Risikogruppe 2 in begrenzten Mengen (<300 kg) gemäß Randnummer 10011 Anlage B ADR (ab 1. Juli 2001 Unterabschnitt 1.1.3.6). Weiterhin sind befreit Einrichtungen (z. B.Labors), die gefährliche Güter nur empfangen.

Unabhängig von einer möglichen Befreiung oder Bestellung eines Gefahrgutbeauftragen sind zur Gewährleistung der Sicherheit beim Transport des o.g. medizinischen Untersuchungsmaterials - auch wenn Erleichterungen wie die Vereinbarung M96 genutzt werden - all die Mitarbeiter als „Beauftragte oder sonstige verantwortliche Person" persönlich verantwortlich und zu schulen, denen entsprechende Aufgaben zur eigenverantwortlichen Erledigung übertragen werden. Dabei handelt es sich insbesondere um das mit der sachgerechten Verpackung und Kennzeichnung betraute Personal und um die Fahrzeugführer der Transportfahrzeuge. Sie sind durch interne oder externe und $\mathrm{zu}$ bescheinigende Schulungen in wiederkehrenden Abständen über die zu beachtenden gefahrgutrechtlichen Vorschriften und ihre praktische Umsetzung zu schulen. Diese Schulungen müssen nicht - wie diejenigen für Gefahrgutbeauftragte - in von der IHK anerkannten Lehrgängen und durch von der IHK zugelassene Schulungsveranstalter durchgeführt werden, sondern können fachbezogen intern oder extern vorgenommen werden, z. B. durch den jeweiligen Gefahrgutbeauftragten. 


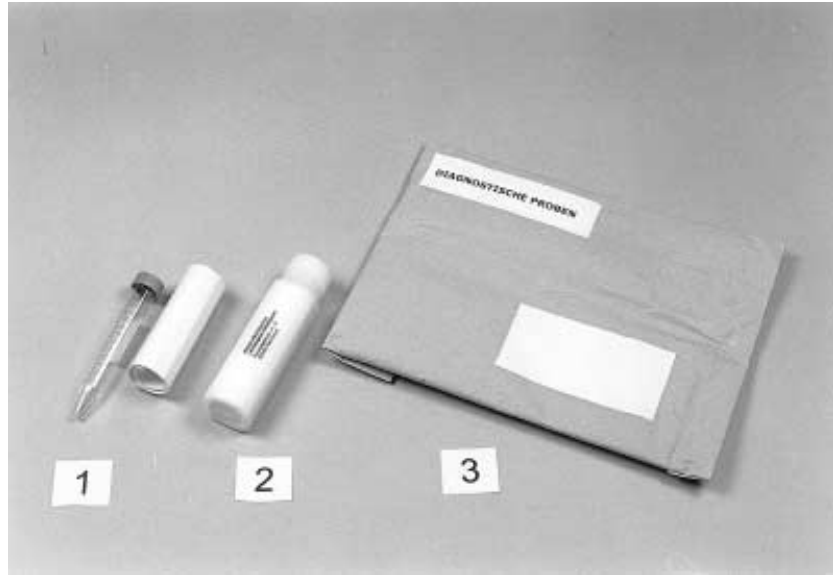

Abb. $2<$ Verpackung für infektiöse Materialien nach der M96 bzw. EN829; 1. Probenröhrchen mit saugfähigem Material, 2. Schutzgefäß, 3. Versandhülle

\section{Verantwortlichkeiten}

Die Hauptverantwortung für die Einhaltung der Sicherheitsvorschriften beim Transport von Gefahrgütern liegt beim Absender. Im speziellen Fall des Versandes von medizinischem oder biologischem Untersuchungsgut durch medizinische Einrichtungen ist dies im Zweifelsfall in persona der Inhaber oder Leiter der betr. Einrichtung und nicht automatisch eine möglicherweise nicht eingewiesene Kraft, die vertretungsweise gerade Proben einpackt.

\section{„Die Hauptverantwortung für die Einhaltung der Sicherheitsvorschriften beim Transport von Gefahrgütern liegt beim Absender."}

Wie aus Vorstehendem ersichtlich, kann der Inhaber oder Leiter der betr. Einrichtung die Verantwortung des Absenders ganz oder teilweise übertragen, z. B. auf einen von ihm bestellten Gefahrgutbeauftragten oder auf ,sonstige verantwortliche Personen“. Das könnte z.B.der hygienebeauftragte Arzt, der Laborleiter oder eine speziell eingewiesene Schwester oder MTA sein. Wichtig ist neben der Zuverlässigkeit die Vermittlung der Sachkunde bei dem mit beförderungsrelevanten Aufgaben betrauten Personal für die Bereiche, in denen dieses jeweils verantwortlich ist. Für den Absender sind dies insbesondere die sachgerechte Klassifizierung in die betr. Risikogruppe und die gewissenhafte Einhaltung der entsprechenden Verpackungs- und Deklarationsvorschriften.

Die Kenntnis und verantwortungsvolle Umsetzung der gefahrgutrechtlichen Vorschriften beim Versand und Transport infektiöser Materialien durch medizinische Einrichtungen ist ein Stück praktizierter Infektionsschutz, um die Gefährdung der damit betrauten Personen und der öffentlichen Sicherheit auf ein gesellschaftlich tolerierbares Maß zu minimieren.

Die Autoren danken Herrn OAR Gregor Oberreuter, Referat A44 des Bundesministeriums für Verkehr, Bau- und Wohnungswesen, für die angenehme und konstruktive fachliche Zusammenarbeit bei der Diskussion und Überarbeitung des Manuskriptes sowie für seine wertvollen Anregungen und Hinweise.

\section{Literatur}

1. United Nations (1999) Recommendations on the transport of dangerous goods - model regulations, Eleventh revised edition. United nation publication, New York Geneve

2. Europäisches Übereinkommen vom 30. Sept. 1957 über die internationale Beförderung gefährlicher Güter auf der Straße (ADR) in der Fassung der Bekanntmachung der Neufassung vom 12. Okt. 1998; Anlagen A und B, BGB 1998 II Nr. 44 mit Anlageband

3. Gefahrgutverordnung Straße (GGVS) (1998) Bekanntmachung der Neufassung der GGVS vom 22.12.1998. BGBI I, S 3993

4. Ordnung über die internationale Eisenbahnbeförderung gefährlicher Güter (RID Rahmenrichtlinie 96/49 EG). ABL der EG Nr. L 235, S 25 vom 17.9.1996
5. ADN-Rahmenrichtlinie vom 16.7.1997. ABL der EG Nr.C267, S 96 vom 3.9.1997

6. Bekanntmachung zum Internationalen Code für die Beförderung gefährlicher Güter mit Seeschiffen (IMDG-Code deutsch) vom 8.12.1998. BAnz. Nr. 245 vom 30.12.1998

7. ICAO Technical Instructions for the Safe Transport of Dangerous Goods by Air, International Civil Aviation Organization. Ausgabe 1999/2000

8. IATA Dangerous Goods Regulations, International Air Transport Association. 41. Ausgabe

9. Verordnung über die Bestellung von Gefahrgutbeautragten und die Schulung der beauftragten Personen in Unternehmen und Betrieben (Gefahrgutbeauftragtenordnung - GbV) vom 12.12.1989 in der Fassung der Bekanntmachung vom 26.03.1998. BGBI I,S 640,zuletzt geändert durch die 2.VO zur Änderung der GbV vom 21.12.1999. BGBI I, S 2509

10. Richtlinie 90/679/EWG des Rates vom 26. November 1990 über den Schutz der Arbeitnehmer gegen Gefährdung durch biologische Arbeitsstoffe bei der Arbeit. ABL der EGL 374 vom 31.12.1990

11. Verordnung über Sicherheit und Gesundheitsschutz bei Tätigkeiten mit biologischen Arbeitsstoffen (Biostoffverordnung - BioStoffv) vom 27.1.1999. BGBI 1999 I Nr.4,S50

12. Merkblatt über die Vermeidung und die Entsorgung von Abfällen aus öffentlichen und privaten Einrichtungen des Gesundheitsdienstes der Länder-Arbeits-Gemeinschaft Abfall (LAGA)

13. Laboratory Biosafety Manual, second edition (1993), World Health Organization (WHO), Eigenverlag

14. Gefahrgut-Ausnahmeverordnung (GGAV) vom 23.6.1993. BGBI I,S 994, zuletzt geändert am 23.06.1999 BGBII,S 1435

15. Multilaterale Sondervereinbarung $M 96$ gemäß Rn 2010 und 10602 ADR - Beförderung von Diagnostischen Proben vom 19.6.2000

16. Allgemeine Geschäftsbedingungen der Deutschen Post AG für den Briefdienst Inland vom 1.10.1999 in Verbindung mit den „Regelungen für die Postbeförderung von gefährlichen Stoffen" (Stand Januar 1999)

17. Regelungen für die Postbeförderung von medizinischem und biologischem Untersuchungsgut im Inland, Tipps für Einlieferer (Stand Februar 1999) der Deutschen Post AG

18. EN 829 1996; European Commitee for Standardization, Brüssel 